\title{
Aldosterone induces renal fibrosis by promoting HDAC1 expression, deacetylating H3K9 and inhibiting klotho transcription
}

\author{
LINGYUN LAI, PING CHENG, MINHUA YAN, YONG GU and JUN XUE
}

Department of Nephrology, Huashan Hospital, Fudan University, Shanghai 200040, P.R. China

Received February 25, 2018; Accepted November 26, 2018

DOI: $10.3892 / \mathrm{mmr} .2018 .9781$

\begin{abstract}
Aldosterone has an important role in the progression of renal fibrosis. In the present study, the concentration of aldosterone and klotho (KL) in the serum of patients with chronic kidney disease (CKD) were analyzed. A negative correlation was observed between aldosterone and KL, suggesting that KL may serve a protective role in CKD. Subsequently, an aldosterone-induced CKD mouse model was established using a single nephrectomy and subcutaneous osmotic pump with aldosterone and $1 \%$ high-salt drinking water. It was demonstrated that fibronectin $1(F n l)$ expression levels were higher in high aldosterone mice, whereas $K L$ expression levels were low. In addition, the results demonstrated that histone deacetylase 1 (HDAC1) protein expression levels were upregulated in the renal distal convoluted tubules of high aldosterone mice, whereas acetylated H3K9 (H3K9Ac) was significantly downregulated. To determine the transcriptional activation status, chromatin immunoprecipitation polymerase chain reaction (PCR) was used to validate binding of H3K9Ac to the $K L$ gene promoter site. It was revealed that the binding product of the $K L$ promoter could be PCR-amplified at the H3K9Ac site from wild-type and low aldosterone mice; however, amplification of the binding product was not observed in high aldosterone mice. In conclusion, aldosterone significantly inhibited $\mathrm{H} 3 \mathrm{~K} 9$ acetylation by upregulating $\mathrm{HDAC} 1$ protein expression levels in the renal distal convoluted tubule cells, resulting in its inability to bind to the $K L$ promoter, loss of transcription of the $K L$ gene and increased expression of the renal fibrosis gene, Fnl.
\end{abstract}

\section{Introduction}

Chronic kidney disease (CKD) is a major disease that threatens human health (1-4), and renal fibrosis is the final pathological

Correspondence to: Professor Jun Xue, Department of Nephrology, Huashan Hospital, Fudan University, 12 Middle Wulumuqi Road, Shanghai 200040, P.R. China

E-mail: xuejun@fudan.edu.cn

Key words: renal fibrosis, aldosterone, klotho, histone deacetylase 1, transcriptional activity manifestation of CKD progression (1-4). Previous reports, and our previous studies, have suggested that aldosterone can regulate water and salt metabolism, and also serves a key role in the progression of renal fibrosis $(5,6)$. However, many studies have only focused on the mechanisms that promote fibrosis (7-11). Klotho (KL) is predominantly expressed in the distal tubules of the kidney and is a cofactor for the fibroblast growth factor 23 receptor (12-14). Previous studies have suggested that KL reduction is closely associated with the progression of CKD fibrosis, as kidney KL expression is reduced in different forms of CKD, including chronic glomerulonephritis, acute renal tubular injury and diabetic nephropathy, leading to the promotion of transforming growth factor $\beta 1$ and the expression of other fibrosis factors (12-20). In addition, renal fibrosis was significantly reduced in neonatal mice that overexpressed $K L(16,19)$. The above studies suggest that, in accelerating CKD progression, aldosterone increase is accompanied by the reduction of KL expression. There may be some association between the changes in these two factor in CKD.

Histone deacetylase (HDACs) are a class of enzymes that remove acetyl groups from histone amino acid residues, and serve an important role in chromatin structural modification and gene transcription regulation (16,21-26). Generally, HDACs can inhibit gene transcription activation by blocking DNA dissociation from histone octamers and tightening the structure of nucleosomes, which inhibits binding between DNA and transcription factors or synergistic transcription regulators (16,21-26).

The current study therefore explored whether high aldosterone significantly reduces acetylation of the $\mathrm{H} 3 \mathrm{~K} 9$ site by activating HDAC1 protein expression in the renal distal convoluted tubule cells, inducing the loss of $K L$ gene transcriptional activity and ultimately leading to the exacerbation of renal fibrosis.

\section{Patients and methods}

Selection of patients with CKD. Adult patients were screened if they had a CKD stage between 1-5, according to the Kidney Disease Outcomes Quality Initiative (KDOQI) criteria published in January 2012 (27). According to the KDOQI criteria, patients are classified into five stages based on their estimated GFR (eGFR). The eGFR was determined by the CKD Epidemiology Collaboration creatinine equation (28). A total of 115 adults were screened, of whom three were excluded 
from the study as they were diagnosed with acute kidney injury. Finally, 112 patients (77 men and 35 women; 31-88 years, mean age $64.5 \pm 12.7$ years) were enrolled and underwent a 6-year follow-up between January 2010 and December 2015 at Huashan Hospital, Fudan University, Shanghai, China.

Blood samples for laboratory measurements were obtained at the time of enrolment and at 1.5-years follow-up. The concentration of serum soluble $\alpha$-KL was determined using an ELISA kits obtained from Immuno-Biological Laboratories Co., Ltd. (cat. no. 27998; Fujioka, Japan), according to the manufacturer's instructions. $\Delta$ aldosterone was calculated using the concentration of aldosterone at baseline and at 1.5-years follow-up ( $\Delta$ value=value at the 1.5-year follow-up-value at the baseline).

The present study was approved by the Ethics Committee of Huashan Hospital, Fudan University (Shanghai, China). All provided written informed consent for participation and the study was conducted in accordance with the Declaration of Helsinki.

Aldosterone-induced CKD mouse model and serum aldosterone detection. A total of 20 adult C57 male mice at 7-8 weeks of age and $20 \mathrm{~g}$ weight, were obtained from the Animal Research Center, Fudan University (Shanghai, China). They were housed at 4 mice per cage and maintained for 14 days in a temperature-controlled environment under a 12-h light/dark cycle with ad libitum access to food and water. The mice were uninephrectomized. Following a 2 week recovery period, an ALZET ${ }^{\circledR}$ osmotic pump (model no. 2002; DURECT Corporation, Cupertino, CA, USA) was implanted subcutaneously (29). Subsequently, mice were divided into three treatment groups: Group 1 , vehicle $(0.5 \%$ ethanol, subcutaneously) $(\mathrm{n}=4)$ as the WT group; Group 2, vehicle (subcutaneously) $+1 \% \mathrm{NaCl}$ in drinking water $(\mathrm{n}=8)$ as the low aldosterone group; Group 3, $0.75 \mu \mathrm{g} / \mathrm{h}$ aldosterone (subcutaneously) $+1 \% \mathrm{NaCl}$ in drinking water $(n=8)$ as the high aldosterone group (29). Mice were treated for 2 weeks. The study was approved by the Ethics Committee of Fudan University (reference no. 201702069S; Shanghai, China). All experiments conformed to standards set by the Laboratory Animal Regulation of the State Scientific and Technological Commission (Shanghai Lab. Animal Research Center).

The concentration of serum aldosterone was ascertained using a chemiluminescent immunoassay obtained from Shenzhen New Industries Biomedical Engineering Co. Ltd. Shenzhen, China (cat. no. 0525-2007).

Hematoxylin and eosin $(H \& E)$ staining. Briefly, fresh kidney tissues were fixed in 4\% paraformaldehyde (Sigma-Aldrich; Merck KGaA, Darmstadt, Germany) for $30 \mathrm{~min}$ at room temperature. Then, the tissue was dehydrated using graded ethanol (70, 80, 90 and 100\%), embedded in paraffin, sectioned (6- $\mu \mathrm{m}$ thickness), and immersed in xylene for dewaxing. The sections were stained with H\&E (Sigma-Aldrich; Merck $\mathrm{KGaA}$ ). The rehydrated sections were stained in hematoxylin solution for $30 \mathrm{~min}$ at room temperature and washed in tap water for $5 \mathrm{~min}$ until the sections turned blue. The sections were differentiated in $70 \%$ ethanol containing $1 \% \mathrm{HCl}$ for 5 seconds and then washed for 5 min in tap water until blue. The sections were then stained in eosin solution for $10 \mathrm{~min}$. The sections were cleared with xylene and sealed with neutral resin (both Sigma-Aldrich; Merck KGaA). The sections were observed under a light microscope at magnification, x100-400 and three fields of view were observed.

Reverse transcription-quantitative polymerase chain reaction $(R T-q P C R)$. Total kidney RNA was extracted from each group of mice using TRIzol ${ }^{\circledR}$ (Invitrogen; Thermo Fisher Scientific, Inc., Waltham, MA, USA). The RNA was processed using DNase I (Sigma-Aldrich; Merck KGaA) and reverse transcribed to cDNA using the ReverTra Ace- $\alpha-{ }^{\circledR}$ kit [Toyobo (Shanghai) Co., Ltd., Shanghai, China]. The RNA and primer Oligo dt were heated in a $70^{\circ} \mathrm{C}$ heat block for 5 min then immediately chilled in ice water for $\geq 5 \mathrm{~min}$, then centrifuged at $15,725 \mathrm{x}$ g for $10 \mathrm{sec}$ in a microcentrifuge at $4^{\circ} \mathrm{C}$ before being stored on ice until reverse transcription mix was added. qPCR was performed using a SYBR ${ }^{\circledR}$ Green Realtime PCR Master Mix [Toyobo (Shanghai) Co., Ltd.] on a MasterCycle RealPlex ${ }^{4}$ real-time PCR detection system (Eppendorf, Hamburg, Germany). The sequences of forward and reverse primers of Klotho was 5'GAGTGGCAT AGGGGCTACA and 5'GGCTGGTTTTCAGGTAAAGG, respectively. The sequences of forward and reverse primers of fibronectin 1 was 5'AGTGGAAGTGTGAGCGACAT and 5'GTGAGTCTGCGGTTGGTAAAT, respectively. The sequences of forward and reverse primers of $18 \mathrm{~S}$ was $5^{\prime} \mathrm{CAG}$ CCACCCGAGATTGAGCA and 5'TAGTAGCGACGGGCG GTGTG, respectively. The thermocycling conditions were as follows: 40 cycles of denaturation at $95^{\circ} \mathrm{C}$ for $15 \mathrm{sec}$, annealing at $58^{\circ} \mathrm{C}$ for $30 \mathrm{sec}$ and extension at $72^{\circ} \mathrm{C}$ for $42 \mathrm{sec}$. The relative expression levels of the gene were determined by the $2^{-\Delta \Delta \mathrm{Cq}}$ method (30). The mRNA expression levels were normalized to the internal reference gene, $18 \mathrm{~S}$ ribosomal RNA.

Immunohistochemical staining. Briefly, fresh kidney tissues were fixed in $4 \%$ paraformaldehyde (Sigma-Aldrich; Merck $\mathrm{KGaA}$ ) for $30 \mathrm{~min}$ at room temperature. Then, the tissue was dehydrated in an ethanol gradient (70, 80, 90 and 100\%), embedded in paraffin, sectioned (6- $\mu \mathrm{m}$ thickness) and immersed in xylene for dewaxing. The sections were blocked using an immunohistochemical blocking solution (Beyotime Institute of Biotechnology, Haimen, China) at $37^{\circ} \mathrm{C}$ for $30 \mathrm{~min}$. After the blocking solution was discarded, the samples were washed in washing buffer (Beyotime Institute of Biotechnology) three times, 5 min each at room temperature. The samples were incubated with antibodies (rabbit anti-mouse HDAC1; cat. no. 34589 and rabbit anti-mouse H3K9Ac; cat. no. 9927, 1:200; Cell Signaling Technology, Inc., Danvers, MA, USA) at $37^{\circ} \mathrm{C}$ for $45 \mathrm{~min}$. The horseradish peroxidase-conjugated secondary antibody (cat. no. sc-2768; 1:200; Santa Cruz Biotechnology, Inc., Dallas, TX, USA) was added and the sections were incubated for $60 \mathrm{~min}$ at room temperature. Finally, a VECTASTAIN Elite ABC kit (cat. no. PK-6100; Vector Laboratories, Inc., Burlingame, CA, USA) was used for the color reaction. Meanwhile, PBS ( $\mathrm{pH}$ 7.4) was used as a negative control in the place of the first antibody. After the antibody was discarded, the samples were washed in washing buffer three times, 5 min each at room temperature. Finally, the samples were mounted in neutral resin (Sigma-Aldrich; Merck KGaA). 
Masson's trichrome staining was used at room temperature for $10 \mathrm{~min}$ to detect collagen fibers in the tissues: Collagen fibers were stained blue, nuclei are stained dark brown and background is stained red. The sections were observed under a light microscope at magnification, x100-400 and three fields of view were observed.

Western blot analysis. Total protein was extracted using NP-40 (Nonidet P-40) lysis buffer over ice and the protein concentration determined with a BCA Protein Assay kit (cat. no. 23225; Thermo Fisher Scientific, Inc.). Protein at $30 \mu \mathrm{g} /$ lane protein was separated by $12 \%$ SDS-PAGE gels and transferred onto polyvinylidene fluoride membranes. The membranes were blocked using $5 \%$ bovine serum albumin (cat. no. 37520; Thermo Fisher Scientific, Inc.) in Tris-buffered saline with Tween-20 or with Triton X-100 and then incubated with primary antibodies overnight at $4^{\circ} \mathrm{C}$. The antibodies of HDAC1 and $\mathrm{H} 3 \mathrm{~K} 9 \mathrm{Ac}$ were the same as used for immunohistochemical staining, dilution 1:500 together with rabbit anti-mouse klotho antibody (cat. no. ab154163; 1:1,000; Abcam, Cambridge, MA, USA). Subsequently, the membranes were incubated with horseradish peroxidase (HRP)-conjugated secondary antibodies (goat anti-rabbit IgG; cat. no. sc-2357; 1:200; Santa Cruz Biotechnology, Inc.; rabbit anti-mouse $\beta$-actin antibody; cat. no. ab8227; 1:2,000; Abcam) for $1 \mathrm{~h}$ in $37^{\circ} \mathrm{C}$. The membranes were washed to remove unbound antibodies and proteins were detected using ECL Immobilon Western Chemiluminescent HRP Substrate (cat. no. WBKLS0500; EMD Millipore, Billerica, MA, USA). The protein bands were visualized using a Kodak XAR-5 film chemiluminescence imaging system (Sigma-Aldrich; Merck KGaA). The protein levels were normalized to the reference protein $\beta$-actin.

Chromatin immunoprecipitation (ChIP). ChIP reactions were performed using the EZ-ChIP ${ }^{\text {Tм }}$ kit (EMD Millipore, Billerica, MA, USA), according to the manufacturer's instructions. Briefly, the mouse kidney samples were fixed in $1 \%$ paraformaldehyde for $30 \mathrm{~min}$ at $37^{\circ} \mathrm{C}$ and then $125 \mathrm{mM}$ glycine was added for $10 \mathrm{~min}$ at room temperature to terminate the cross-linking. Ultrasonication was used to fragment the DNA into 200-1,000 bp chromatin fragments. The sonicator was adjusted to $30 \%$ intensity and $2 \mathrm{~mm}$ pulse wave, 50 watts of power. All samples received 3 sonications for $10 \mathrm{sec}$ each, $15 \mathrm{sec}$ apart. The chromatin fragments were incubated overnight at $4^{\circ} \mathrm{C}$ with primary antibodies (Rabbit anti-mouse H3K9Ac; cat. no. 9649; 1:100; Cell Signaling Technology, Inc.). Then, Pierce ${ }^{\mathrm{TM}}$ Protein A/G Plus Agarose (Thermo Fisher Scientific, Inc.) was added to the solution to obtain immunoprecipitated chromatin for PCR analysis. The PCR conditions were as follows: Denaturation at $95^{\circ} \mathrm{C}$ for $30 \mathrm{sec}$, annealing at $55^{\circ} \mathrm{C}$ for $30 \mathrm{sec}$ and extension at $72^{\circ} \mathrm{C}$ for $30 \mathrm{sec}$ for 30 cycles. The ChIP PCR $K L$ promoter primers were as follows: Forward, 5'-CTCAGGATGGAG GCCACAGG-3' and reverse, 5'-CACAGCAGGGGTCATAGG GA-3'. The amplified product was visualized on a $1.2 \%$ agarose gel. The ChIP PCR product was on the $K L$ promoter region from -215 to $-134 \mathrm{bp}$.

Statistical analysis. Statistical analyses were performed using GraphPad Prism software version 5.0 (GraphPad Software, Inc., La Jolla, CA, USA) or SPSS software version 17.0
(SPSS, Inc., Chicago, IL, USA). The data are presented as the mean \pm standard error. Differences among multiple groups were evaluated using one-way analysis of variance and differences between two groups were evaluated using Student's t test. Correlation between two continuous variables was determined using Pearson's correlation coefficient. $\mathrm{P}<0.05$ was considered to indicate a statistically significant difference.

\section{Results}

High aldosterone levels downregulate KL and upregulate fibronectin 1 (Fnl) in mice with CKD. Blood samples were collected from 112 patients with CKD, and the serum KL and aldosterone concentrations were determined. Correlation analysis indicated that the serum $\Delta$ aldosterone at 1.5 -years follow-up was significantly negatively correlated with serum $\mathrm{KL}(\mathrm{R}=-0.237, \mathrm{P}<0.01$; Fig. 1A).

In the aldosterone-induced CKD mouse model, aldosterone levels in the peripheral blood of mice in the high aldosterone group $(34,702.01 \pm 4,307.62 \mathrm{pmol} / 1)$ were significantly higher compared with the low aldosterone $(462.19 \pm 148.87 \mathrm{pmol} / \mathrm{l})$ and wild-type (WT) groups (656.11 $\pm 32.76 \mathrm{pmol} / \mathrm{l}$; Fig. 1B).

qPCR measurement of $K L$ and $F n l$ demonstrated that $K L$ mRNA expression levels were significantly lower, whereas $F n l$ expression levels were significantly higher in the renal tubules of high aldosterone mice compared with low aldosterone and WT mice (Fig. 1C). The western blot results were consistent and revealed a significantly lower KL protein expression in the kidneys of high aldosterone mice compared with low aldosterone and WT mice (Fig. 1D). These results suggested that KL expression was inversely proportional to aldosterone release and renal fibrosis.

High aldosterone levels induce histone deacetylation in the distal convoluted tubules of mice with CKD. Immunohistochemical staining revealed that HDAC1 expression was absent in the distal convoluted tubules of WT mice and HDAC1 expression was weak in low aldosterone mice. However, HDAC1 expression was markedly increased in high aldosterone mice. In addition, H3K9Ac was strongly expressed in the distal convoluted tubules of WT and low aldosterone mice, whereas H3K9Ac staining was absent in high aldosterone mice (Fig. 2). Western blotting demonstrated that HDAC1 was highly expressed and H3K9Ac was rarely modified in the kidneys of high aldosterone mice (Fig. 3A). The results indicated that high aldosterone levels induced renal injury, resulting in high HDAC1 expression in the distal convoluted tubules and $\mathrm{H} 3 \mathrm{~K} 9$ deacetylation.

High aldosterone levels inhibit transcription of the $K L$ gene in the distal convoluted tubules of mice with CKD. To determine the transcriptional activation status between $\mathrm{KL}$ and H3K9Ac, ChIP-PCR was used to confirm the binding of the promoter region of $K L$ gene to $\mathrm{H} 3 \mathrm{~K} 9 \mathrm{Ac}$ site. The binding site of the $K L$ promoter region is between 215 and -134 bp (Fig. 3B). The results showed that the binding product of $K L$ promoter was obtained in the H3K9Ac site in the distal convoluted tubules of normal mice and low aldosterone mice (Fig. 3C). However, in high aldosterone mice there was rarely 
A

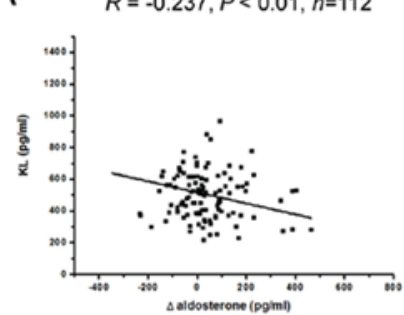

B

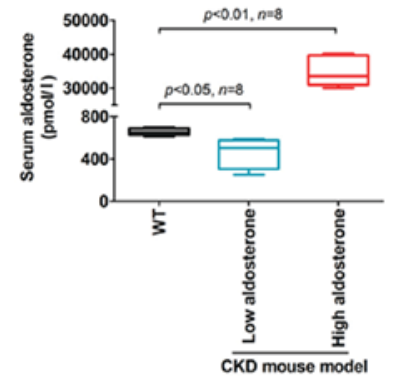

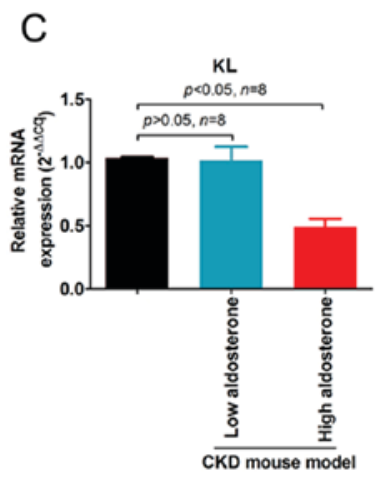
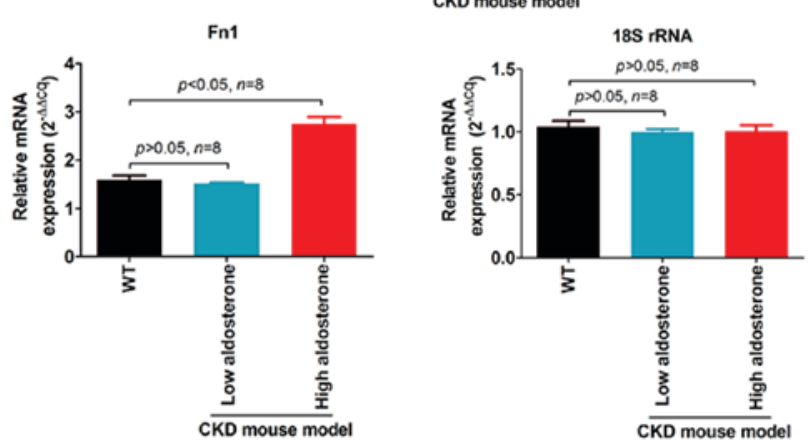

D

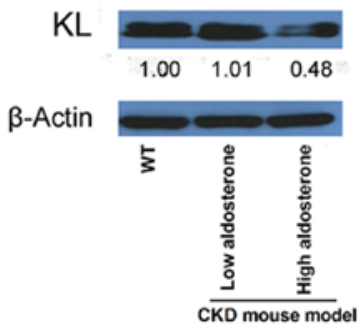

Figure 1. KL expression is inversely proportional to aldosterone release. (A) Serum aldosterone and KL concentrations from 112 patients with CKD followed-up for 1.5 years were significantly negatively correlated. (B) ELISA revealed significantly higher concentrations of aldosterone in the serum of high aldosterone mice compared with low aldosterone and WT mice. (C) Reverse transcription-quantitative polymerase chain reaction demonstrated that $K L$ mRNA, both are normalized to $18 \mathrm{~S}$ RNA, expression levels were significantly lower in the kidney of the high aldosterone mice compared with in low aldosterone and WT mice; whereas the opposite was observed for $F n 1$ mRNA expression levels. (Since in the kidney klotho is mostly expressed in the renal distal convoluted tubules, the above results could reflect the role of aldosterone plays on the expression of klotho in the renal distal convoluted tubules). (D) Western blotting revealed lower KL protein expression levels in the renal distal convoluted tubules of the high aldosterone mice compared with in low aldosterone and WT mice. KL, klotho; WT, wild-type; CKD, chronic kidney disease; Fn1, fibronectin 1; 18S rRNA, 18S ribosomal RNA.

a binding product of $K L$ promoter at the H3K9Ac site. Thus, the results demonstrated that high aldosterone inhibits the acetylation of the $\mathrm{H} 3 \mathrm{~K} 9$ site, leading to its inability to bind to the $K L$ promoter and ultimately inhibits the transcription of the $K L$ gene.

\section{Discussion}

Several studies have reported that high aldosterone can induce renal fibrosis (5) and that KL downregulation promotes CKD fibrosis progression (11). However, the mechanism that leads to $\mathrm{KL}$ downregulation when aldosterone is high remains unclear.

In the present study, $K L$ expression in a high aldosterone-induced CKD mouse model was investigated. KL expression was significantly downregulated by high aldosterone at the mRNA and protein level. The significant differences between the $K L$ mRNA expression levels of the high aldosterone and control groups suggested that aldosterone may regulate $K L$ transcriptional activity to induce $\mathrm{KL}$ mRNA and protein expression.
In addition, it was identified that histone acetylation was different between the high aldosterone and control groups. The results demonstrated that the $\mathrm{H} 3 \mathrm{~K} 9$ locus was deacetylated in the renal tissues of the high aldosterone group. According to epigenetic theory, histone acetylation is necessary to promote transcriptional activation of genes and is maintained by histone acetyltransferases (HAT). Conversely, gene transcription is inhibited when the specific lysine residue at the end of the histone undergoes deacetylation. In general, histone acetylation causes DNA and histone octamer dissociation and nucleosome structure relaxation, enabling gene transcription activation by the specific binding between DNA binding sites and various transcription factors or synergistic transcription regulators. Deacetylated transferases have the opposite effect. In the nucleus, histone acetylation and deacetylation are in dynamic balance and coordinated by HATs and HDACs. HATs transfer the acetyl group of acetyl coenzyme A to the amino-terminal lysine residue of the histone, whereas HDAC deacetylates the histone, binds closely to the negatively charged DNA, yielding a dense structure, which inhibits gene transcription. Based on the above theory, HDAC1 expression was detected in the 


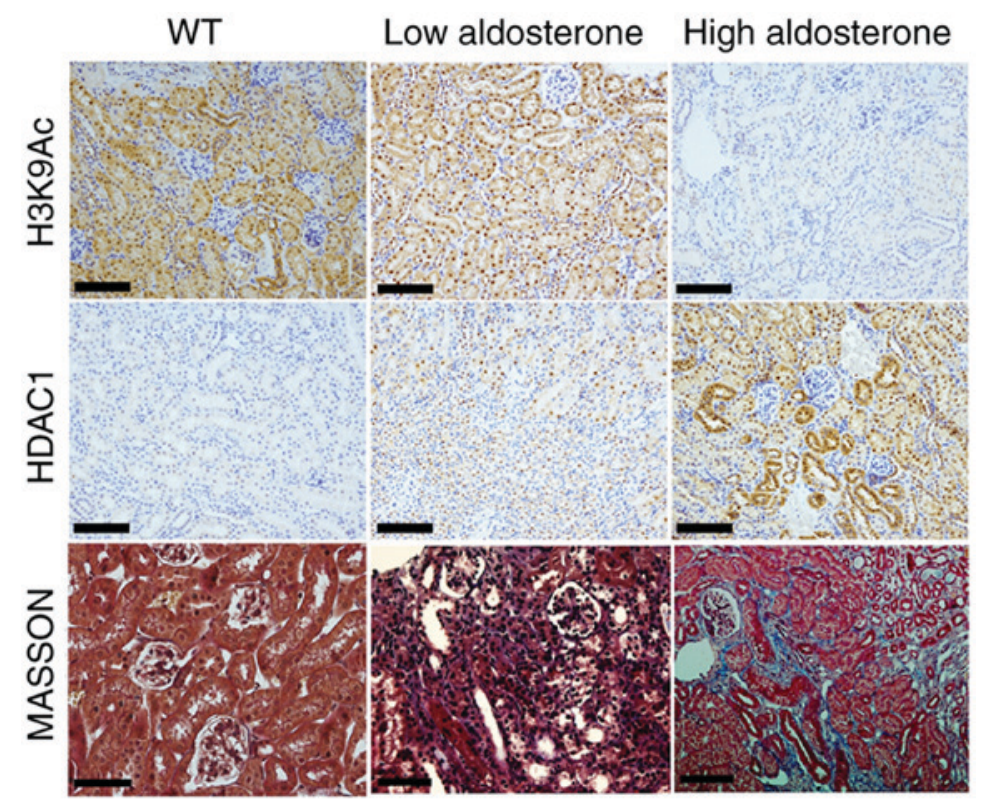

Figure 2. Increased histone deacetylation in the renal distal convoluted tubules of high aldosterone CKD mice. Immunohistochemistry was used to detect histone acetylation and deacetylase in kidneys of CKD model mice. Top: H3K9Ac was increased in the distal convoluted tubules of the WT and low aldosterone mice. However, H3K9Ac was absent in the distal convoluted tubules of the high aldosterone mice. Middle: HDAC1 expression was absent in the distal convoluted tubules of WT mice and low in low aldosterone mice. HDAC1 expression was increased in the distal convoluted tubules of high aldosterone mice. Bottom: Masson trichrome staining revealed that high aldosterone mice exhibited increased kidney fibrosis compared with the low aldosterone and WT mice. Scale bar, $20 \mu \mathrm{m}$. CKD, chronic kidney disease; WT, wild-type; H3K9Ac; acetylated H3K9; HDAC1, histone deacetylase 1.

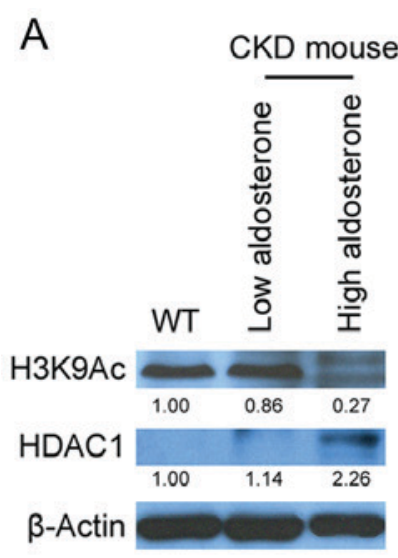

B

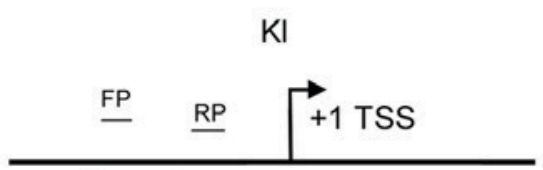

Promoter
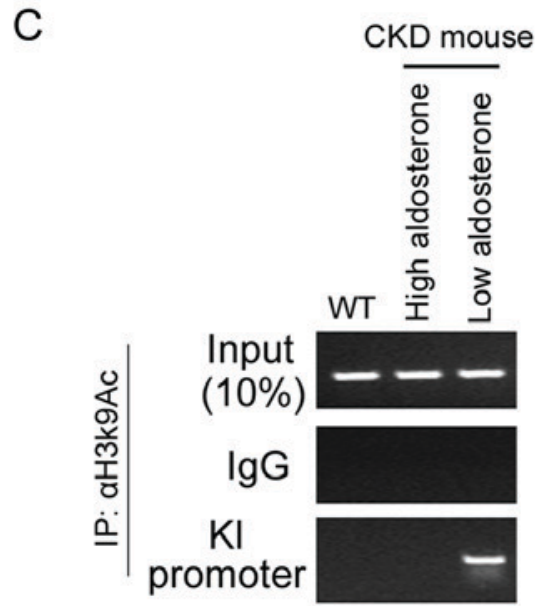

Figure 3. High aldosterone inhibits $K L$ gene transcription in the distal convoluted tubules of CKD mice. (A) Western blot analysis revealed high HDAC1 protein expression levels and low H3K9Ac expression levels in the distal convoluted tubules of high aldosterone mice. (B) Schematic diagram demonstrating ChIP PCR verification of H3K9Ac binding to the $K L$ gene promoter region, to determine transcriptional activation of the $K L$ gene. (C) ChIP qPCR revealed presence of $\mathrm{H} 3 \mathrm{~K} 9 \mathrm{Ac}$ at the $K L$ promoter in kidney of the low aldosterone mice. The $K L$ promoter binding product was absent in high aldosterone mice. ChIP PCR, chromatin immunoprecipitation polymerase chain reaction; WT, wild-type; CKD, chronic kidney disease; H3K9Ac, acetylated H3K9; HDAC1, histone deacetylase 1; FP, forward primer; RP, reverse primer; KL, klotho; TSS; transcription start site; IgG, immunoglobulin G.

current study. HDAC1 expression was significantly increased in the kidney tissues of high aldosterone mice. Based on this result, it can be hypothesized that histone deacetylation in the renal tissue of mice with CKD may inhibit transcription of the $K L$ gene during high aldosterone levels. Subsequently, ChIP was used to analyze the acetylation of the H3K9 site associated with the $K L$ gene promoter. The experimental demonstrated deacetylation of the H3K 9 site associated with the $K L$ gene promoter under high aldosterone.

In conclusion, the present study demonstrated that high aldosterone increased HDAC1 expression levels in the distal convoluted tubules in CKD mice, leading to histone H3K9 deacetylation, inhibition of $K L$ gene transcription. As a previous study demonstrated the role of increasing aldosterone in renal damage (31), the above results may explain why klotho decreased in CKD and may help lead to renal fibrosis.

\section{Acknowledgements}

The authors would like to thank Ms. Huizhu Shi (Department of Nephrology, Huashan Hospital, Fudan University) for her assistance with collection of patient samples. 


\section{Funding}

The present study was supported by National Natural Science Foundation of China (grant no. 81670697).

\section{Availability of data and materials}

All data generated or analyzed during this study are included in this published article.

\section{Authors' contributions}

JX and LL designed the study and participated in revising the manuscript. LL and YG followed-up the patient cohort in the CKD clinic. LL, MY and PC performed the experiments and statistical analyses. LL drafted the manuscript. All authors read and approved the final manuscript.

\section{Ethics approval and consent to participate}

The present study was approved by the Ethics Committee of Huashan Hospital, Fudan University (Shanghai, China). All provided written informed consent for participation and the study was conducted in accordance with the Declaration of Helsinki.

\section{Patient consent for publication}

All patients in this cohort agreed their data for publication.

\section{Competing interests}

The authors declare that they have no competing interests.

\section{References}

1. Allison SJ: Chronic kidney disease: The effect of age on CKD outcomes. Nat Rev Nephrol 9: 3, 2013.

2. Hallan SI, Matsushita K, Sang Y, Mahmoodi BK, Black C, Ishani A, Kleefstra N, Naimark D, Roderick P, Tonelli M, et al: Age and association of kidney measures with mortality and end-stage renal disease. JAMA 308: 2349-2360, 2012.

3. Kovesdy CP, Alrifai A, Gosmanova EO, Lu JL, Canada RB, Wall BM, Hung AM, Molnar MZ and Kalantar-Zadeh K: Age and outcomes associated with $\mathrm{BP}$ in patients with incident $\mathrm{CKD}$. Clin J Am Soc Nephrol 11: 821-831, 2016.

4. Tangri N, Komenda P and Rigatto C: Age and outcomes in CKD. Am J Kidney Dis 62: 225-227, 2013.

5. Lai L, Chen J, Hao CM, Lin S and Gu Y: Aldosterone promotes fibronectin production through a Smad2-dependent TGF-beta1 pathway in mesangial cells. Biochem Biophys Res Commun 348: 70-75, 2006.

6. Brown NJ: Contribution of aldosterone to cardiovascular and renal inflammation and fibrosis. Nat Rev Nephrol 9: 459-469, 2013.

7. Herrada AA, Contreras FJ, Marini NP, Amador CA, González PA, Cortés CM, Riedel CA, Carvajal CA, Figueroa F, Michea LF, et al: Aldosterone promotes autoimmune damage by enhancing Th17-mediated immunity. J Immunol 184: 191-202, 2010.

8. Huby AC, Antonova G, Groenendyk J, Gomez-Sanchez CE, Bollag WB, Filosa JA and Belin de Chantemèle EJ: Adipocyte-derived hormone leptin is a direct regulator of aldosterone secretion, which promotes endothelial dysfunction and cardiac fibrosis. Circulation 132: 2134-2145, 2015.

9. Mayyas F, Alzoubi KH and Van Wagoner DR: Impact of aldosterone antagonists on the substrate for atrial fibrillation: Aldosterone promotes oxidative stress and atrial structural/electrical remodeling. Int J Cardiol 168: 5135-5142, 2013.
10. McGraw AP, Bagley J, Chen WS, Galayda C, Nickerson H, Armani A, Caprio M, Carmeliet P and Jaffe IZ: Aldosterone increases early atherosclerosis and promotes plaque inflammation through a placental growth factor-dependent mechanism. J Am Heart Assoc 2: e000018, 2013.

11. Zhang M, Chen J, Lai L, You L, Lin S, Hao C and Gu Y: Aldosterone promotes fibronectin synthesis in rat mesangial cells via ERK1/2-stimulated $\mathrm{Na}-\mathrm{H}+$ exchanger isoform 1 . Am J Nephrol 31: 75-82, 2010.

12. Bleskestad IH, Thorsen IS, Jonsson G, Skadberg Ø, Bergrem H and Gøransson LG: Soluble Klotho and intact fibroblast growth factor 23 in long-term kidney transplant patients. Eur J Endocrinol 172: 343-350, 2015.

13. Haruna Y, Kashihara N, Satoh M, Tomita N, Namikoshi T, Sasaki T, Fujimori T, Xie P and Kanwar YS: Amelioration of progressive renal injury by genetic manipulation of klotho gene. Proc Natl Acad Sci USA 104: 2331-2336, 2007.

14. Lu X and Hu MC: Klotho/FGF23 axis in chronic kidney disease and cardiovascular disease. Kidney Dis (Basel) 3: 15-23, 2017.

15. Drew DA, Katz R, Kritchevsky S, Ix J, Shlipak M, Gutiérrez OM, Newman A, Hoofnagle A, Fried L, Semba RD and Sarnak M: Association between soluble Klotho and change in kidney function: The health aging and body composition study. J Am Soc Nephrol 28: 1859-1866, 2017.

16. Lin W, Li Y, Chen F, Yin S, Liu Z and Cao W: Klotho preservation via histone deacetylase inhibition attenuates chronic kidney disease-associated bone injury in mice. Sci Rep 7: 46195, 2017.

17. Park MY, Herrmann SM, Saad A, Eirin A, Tang H, Lerman A, Textor SC and Lerman LO: Biomarkers of kidney injury and klotho in patients with atherosclerotic renovascular disease. Clin J Am Soc Nephrol 10: 443-451, 2015.

18. Ritter CS, Zhang S, Delmez J, Finch JL and Slatopolsky E: Differential expression and regulation of Klotho by paricalcitol in the kidney, parathyroid, and aorta of uremic rats. Kidney Int 87: 1141-1152, 2015.

19. Zhang Q, Liu L, Lin W, Yin S, Duan A, Liu Z and Cao W: Rhein reverses Klotho repression via promoter demethylation and protects against kidney and bone injuries in mice with chronic kidney disease. Kidney Int 91: 144-156, 2017.

20. Zhou L, Mo H, Miao J, Zhou D, Tan RJ, Hou FF and Liu Y: Klotho ameliorates kidney injury and fibrosis and normalizes blood pressure by targeting the Renin-Angiotensin system. Am J Pathol 185: 3211-3223, 2015.

21. Brocks D, Schmidt CR, Daskalakis M, Jang HS, Shah NM, Li D, Li J, Zhang B, Hou Y, Laudato S, et al: DNMT and HDAC inhibitors induce cryptic transcription start sites encoded in long terminal repeats. Nat Genet 49: 1052-1060, 2017.

22. Cantley MD, Zannettino ACW, Bartold PM, Fairlie DP and Haynes DR: Histone deacetylases (HDAC) in physiological and pathological bone remodelling. Bone 95: 162-174, 2017.

23. Guerriero JL, Sotayo A, Ponichtera HE, Castrillon JA, Pourzia AL, Schad S, Johnson SF, Carrasco RD, Lazo S, Bronson RT, et al: Class IIa HDAC inhibition reduces breast tumours and metastases through anti-tumour macrophages. Nature 543: 428-432, 2017.

24. Hull EE, Montgomery MR and Leyva KJ: HDAC inhibitors as epigenetic regulators of the immune system: Impacts on cancer therapy and inflammatory diseases. Biomed Res Int 2016: 8797206, 2016.

25. Kwon DY, Zhao YT, Lamonica JM and Zhou Z: Locus-specific histone deacetylation using a synthetic CRISPR-Cas9-based HDAC. Nat Commun 8: 15315, 2017.

26. Thoma C: Kidney cancer: Combination of HDAC inhibitor with IL-2 promising. Nat Rev Urol 14: 639, 2017.

27. Inker LA, Astor BC, Fox CH, Isakova T, Lash JP, Peralta CA, Kurella Tamura M and Feldman HI: KDOQI US commentary on the 2012 KDIGO clinical practice guideline for the evaluation and management of CKD. Am J Kidney Dis 63: 713-735, 2014.

28. Levey AS and Stevens LA: Estimating GFR using the CKD Epidemiology Collaboration (CKD-EPI) creatinine equation: More accurate GFR estimates, lower CKD prevalence estimates, and better risk predictions. Am J Kidney Dis 55: 622-627, 2010.

29. Wang B, Ding W, Zhang M, Li H and Gu Y: Rapamycin attenuates aldosterone-induced tubulointerstitial inflammation and fibrosis. Cell Physiol Biochem 35: 116-125, 2015.

30. Livak KJ and Schmittgen TD: Analysis of relative gene expression data using real-time quantitative PCR and the 2(-Delta Delta C(T)) method. Methods 25: 402-408, 2001.

31. Shavit L, Lifschitz MD and Epstein M: Aldosterone blockade and the mineralocorticoid receptor in the management of chronic kidney disease: Current concepts and emerging treatment paradigms. Kidney Int 81: 955-968, 2012. 